\title{
ICHALEC Improved Cluster Head And Low Energy Consumption Protocol In Wireless Sensor Network
}

\author{
Gurpreet Kaur Khalsa ${ }^{1}$,Sachi Pandey ${ }^{2}$ \\ ${ }^{I}$ (Pursuing M-Tech, Department Of Computer Science \& Engineering,SRM University,India) \\ ${ }^{2}$ (Asst. Professor ,Department Of Computer Science \& Engineering,SRM University,India)
}

\begin{abstract}
Wireless Sensor Network is an emerging field for research in most of the aspects of mankind. It is a network composed of nodes without any central controller. Sensor network protocols have high ability to be used in each and every field of life. The working of sensor networks depends entirely on the battery power of sensors which cannot be replaced. In this work, cluster based routing protocol is studied precisely. Further leach protocol is modified as ICHALEC protocolby introducing two schemes efficient cluster head replacement scheme and two power level scheme for amplification of signals. The performance of ICHLEAC protocol is shown in terms of network life time and throughput. The cluster head selection in ICHALEC is more efficient than other protocols because energy is not wasted to select the new cluster head for new round and in other scheme two levels of power are used for amplification of signals either low or high power. Thus using different energy for different types of communication. Then at the end ICHALEC is compared with LEACH and MODLEACH protocol. ICHALEC outperforms LEACH and MOD-LEACH in terms of network lifetime.
\end{abstract}

Keywords: Clusters, ICHALEC, LEACH, MOD-LEACH, Power level, Threshold.

\section{Introduction}

WSN is a large network of nodes which are interconnected by a communication network. These nodes are autonomous objects which are randomly deployed in any region according to the requirement. The number of sensor nodes in a network can vary from some hundreds of nodes to thousands depending on the requirement of the region. A sensor network consist of low power nodes and low cost multifunctional nodes. These sensor nodes communicate via wireless medium over certain distances. The sensor nodes consist of sensing, processing, power and communication component. Each and every component is having its own functions to perform. The arrangement of these sensor nodes is defined in two ways one in deterministic way and other self organizing capability .It means that in deterministic way the routes for data transmission is already defined and sensors are placed manually. Whereas in self organizing way the nodes are scattered randomly in an ad hoc manner .As the energy consideration is also important for setting up the routes. They can provide high quality and fault tolerant network capability [1][2],however there battery lifetime is limited. Ranging from medical applications to military applications WSN is used. The number of nodes in network are potentially large . One of the main important application of the WSN is to monitor the data of the environment and then further transmit that data to the central point called sink node. In order to initiate some specific action the data is analyzed .That data is analyzed by the sink node or by the network. The most important criteria in wireless sensor network is that large number of sensors should be used and the energy consumption of these sensors should be low so that energy is not wasted.

The most prominent limitation in Wireless Sensor Networks is energy limitation constraint. The number of nodes deployed in any region are high so the monitoring of suchnodes becomes very difficult. Once the network is established the node energy keeps on dissipating whenever the information is received or send to other node in network. As the sensor nodes are of small size due to which big size batteries cannot be embedded in them and it is very difficult for the people to replace such small sized batteries .So sensors need efficient mechanism for energy utilization. As sensor networks have dynamic nature so nodes can lose its connection in any network. So each node is tightly power constrained. Therefore the entire network has limited lifetime. In order to enhance the network lifetime of network many routing protocol approaches are used. Thus routing protocol is an important factor affecting the energy consumption of the sensor nodes. One of techniques to enhance network lifetime is clustering. Clustering divides the nodes into separate clusters .In this the large data coming from different nodes is compresses by using the techniques of aggregation. Therefore, only those packets are transmitted which consist meaningful information, this approach leads to an efficient solution for energy. Data aggregation can be executed with the help of clustering technique[3][4]. In any wireless network for efficientperformance, the protocols should be efficient. Many protocols have been designed which address the power problem. One of the most prominent algorithms which are designed to address power issue is direct transmission algorithms, cluster based algorithms and hop to hop transmission algorithms. In further sections, the work done on cluster based routing in wireless sensor network is discussed and some modifications are made 
in existing protocol to increase efficiency. Finally, experiments are made to show the network lifetime and throughput.

\section{Related Work}

In a survey on routing protocols in WSN, it classifies the routing techniques based on network structure into three categories flat based, location based and hierarchical based. Further also these protocols are classified into QoS based, data centric based, mobility based, query based, negotiation based and multipath based protocols. Manufacturing of small nodes capable of transmitting and processing are available nowadays, but hundred of nodes are deployed for any application in any region. These nodes deployed have limited power so the power capability of the nodes should not be wasted. It means the power capability should be used in a précised manner so that it is not wasted and the network lifetime is enhanced. Although efficient circuit is also an essential requirement for energy efficiency but protocols also play an important role in energy consideration and power management.

To deal with such energy problems direct transmission approach was discussed[5].In direct transmission the node senses data from the environment and directly transmits it to the base station. This method is very much efficient for the security measures because there is direct transmission but one drawback of this approach is the excessive power consumption of the nodes. As the nodes which are far away from the base station die out quickly because they require extra power consumption. To solve this problem further protocols are classified in two classes taken into consideration the energy aware broadcast/multicast problems. The algorithm is classified in the MEB/MEM(minimum energy broadcast/multicast)problem and MLB/MLM(maximum lifetime broadcast )problem in wireless networks. Two main energy metrics that are considered in this approach are minimizing the total power consumption of all the nodes involved in multicast session and maximize the operation time until the power of the one node battery is depleted in multicast session.

In order to solve the issues in direct transmission another approach Least transmission energy came into existence. In this the transmission of the data takes place along the network through multi hop. Both direct transmission and least transmission energy lead to same energy problem but the only difference between two was that in least transmission the nodes which are far away from the base station live longer as compare to the nodes which are closer to the base station. The reason for the nearer nodes to die is because all data is routed to base station. However the transmitting most of the data from each node uses much energy. To address this problem Direct Diffusion method is used which involves the data processing and data dissemination[6] .This concept is used in hierarchical clustering mechanism and thus presented clustering based routing protocol[7].Clustering is one of the efficient mechanism in energy conservation of wireless sensor network. Clustering is useful in data query and broadcast of message [8][9]. In sensor network nodes establish clusters and each node nominates one node as cluster head. The node which is chosen as a cluster head is having maximum energy. It is the cluster head which collects the data from each cluster, aggregates the received data and then transmits it to the base station. In this way lifetime of the network is maximized . Whereas some clustering protocols work on the fact that any node which is in the network can be elected as a cluster head.Considering clustering mechanism various protocols are developed and each of the protocol have its own functions[10].Stable Election Protocol(SEP) gives each node a weighted probability of becoming a cluster head[11].The sensor network architecture of Threshold sensitive Energy Efficient sensor network protocol(TEEN) is based on hierarchical grouping where the nodes which are closer to each other form clusters and process goes on until second level is reached.The Adaptive Threshold Energy Efficient sensor network protocol(APTEEN) architecture is same as TEEN .In APTEEN first the previous data is analyzed, then snapshot view of the network is taken and for a specific period of time event is monitored. Power efficient Gathering in Sensor Information Systems (PEGASIS)a chain is formed among the nodes and so that each node can receive and transmit the data, then the collected data moves from node to node and destination node transmits it to the base station.LEACH is among the most popular protocols for the sensor network.Leach[12]protocol proposes that each node should elect itself a cluster head,therefore first $\mathrm{CH}$ selection process is initiated. In Leach protocol each node is assumed to have powerful energy to reach the nearest cluster head. In this nodes transmit to cluster head and cluster head aggregate the data and transmit it to the base station. But using powerful energy for transmission waste energy of the network. Based on LEACH and SEP several numerous protocols are proposed Q-Leach optimize the network life time of homogenous network[13].

\section{Leach Protocol}

Leach is one ofthe prominent proactive sensor network protocol. In leach once the cluster head is chosen, it is responsible for broadcasting message in assigned time schedule. As the cluster head broadcasts a TDMA schedule for the transmission of the data in given order.Each and every node is having its own time slot in the frame during which the transmission takes place. When the last node completes its transmission then the 
schedule is repeated. Leach is a cluster based protocol in which cluster head rotates randomly to evenly distribute the energy among the nodes in the network[14]. Leach protocol works in various rounds. Each round consist of two phases, Set up phase and Steady phase.

1.Setup Phase-In this phase each node decides whether or not to become cluster head. It depends on decision made by the node by choosing a random number between 0 and 1[15]. The node which is having maximum energy is chosen as a cluster head. Then the cluster head $\mathrm{CH}$ broadcasts its message to all clusters that it has become the new cluster head $\mathrm{CH}$. Then all the nodes have to join the cluster, thus all nodes send join request message to the cluster. The nodes join that cluster which is having signal intensity higher. In this way the each $\mathrm{CH}$ know its members. Then $\mathrm{CH}$ creates time schedule so that each node know its time slot during which the data is transmitted.

2.Steady Phase-. In this phase the data is send to the cluster head by there nodes in the allocated time slot. Then cluster head $\mathrm{CH}$ aggregates the data and sends it to the base station BS. The nodes turn radio on only when it senses some important information. But the cluster head has to keep its receiver on so that it can receive the data from the nodes. The routing topology of leach is shown below in fig1

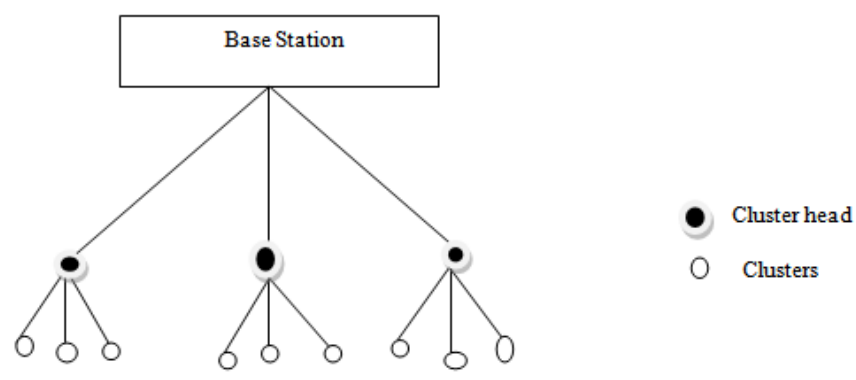

Fig.1 LEACH Routing Topology

The stochastic $\mathrm{CH}$ selection algorithm is used in LEACH. Leach is proposed for homogenous network. When $\mathrm{CH}$ performs its function of aggregation and transmitting, $\mathrm{CH}$ consumes extra energy. Because the node selected as the cluster head dies out quickly due to extra burden. Thus there should be equal distribution of load among all nodes. For this purpose Leach proposes that every node becomes cluster head $\mathrm{CH}$ after $1 / \mathrm{p}$ rounds[16].Each sensor node $n$ generates a random number. And this random number is compared to threshold value $T(n)$. If random number is less then threshold value then sensor node becomes cluster head for that particular round. Otherwise becomes cluster member for that round.

$\mathrm{T}(\mathrm{n})=\mathrm{p} 1-\mathrm{p}(\mathrm{r} \bmod 1 / \mathrm{p}) \quad$ if $\mathrm{n} € \mathrm{G} \quad(1)$

In this formula $\mathrm{p}$ is the probability of the node to be elected as a cluster head. $\mathrm{r}$ is the number of rounds selection. $\mathrm{G}$ is the set of nodes which are not elected as a cluster head in the given round.

\section{Proposed Scheme}

In proposed method two schemes are used Cluster head replacement scheme and two power level scheme for amplification. Basically Leach protocol is modified and in Leach protocol the node elected as cluster head for one round does not becomes cluster head for other rounds. In this way if the cluster head has some energy left during transmission, that energy is wasted. Because the same node cannot become cluster head in next round. Whereas in the proposed scheme if the cluster head has some energy left during its time period and the energy left is greater than the threshold then same cluster head can be chosen for the next round. In this way the energy is not wasted as in Leach. If the cluster head has less energy than the threshold, then new node can be chosen as a cluster head replacing the old one. In second scheme the two different power levels are introduced for the amplification of signals. In intra cluster communication, transmission takes place within cluster and energy required should be less. Whereas in inter cluster communication , where the transmission takes place within the cluster heads the energy requirement is more. So using same energy for both types of transmission is wastage of energy. In this scheme different power levels are used for the different type of transmissions. In this scheme when node becomes cluster head, routing protocol informs the same node to use the high amplification energy and when the same node becomes the cluster member in another round the protocol informs it to switch it to low power level. Finally soft and hard thresholds are applied on this modified Leach.

The operation of new protocol is divided in two phases one full transmission and half transmission . The node selected as a cluster head performs the main functions. The remaining nodes in the cluster broadcast their status to the neighboring nodes. Any node which seems ready to accept this status saves this information and broadcast this message to other nodes that the nodes status indicates that this node can be elected as a new 
cluster head. In this way confirmation message is send to the nodes. The nodes which receive the confirmation message send back the acknowledgement to the cluster head with another confirmation of electing that node to become the cluster head. Thus the cluster head saves the id of that node which is approved to become the cluster head for the next round. In this way the old cluster head receives the data, aggregates the data and then send this data to the base station. If the status of the node which is chosen as the next cluster head shows that this node has high energy to be used as a cluster head in next round as well. Then it is called full transmission .

Whereas in half transmission, only the sensors with cluster head, will aggregate the data and send the data to their respective cluster head.Then the old cluster head will aggregate the data and transmit it to the base station.

\section{Proposed Protocol}

In this section new protocolICHALEC is described.

Cluster head selection:- In this modified protocol ,in the beginning of each round cluster head is elected . In order to determine the ability of the node to become cluster head, random number is generated between 0 and 1. Then is number is compared with the sensor threshold value $T(n)$. If the threshold value is greater then the random number then the node becomes the cluster head for the current round. This random number is actually a sensor residual energy. This energy is generated by the sensor node to determine the method to check the capability of the node to become cluster head. The threshold value for desired cluster head percentage $p$, for sensor $\mathrm{s}$ in round $\mathrm{r}$, with residual energy $\mathrm{R}$ and maximum energy $\mathrm{M}$ is calculated as. $\mathrm{G}$ is a set of nodes which has not been selected as cluster head in last $1 / \mathrm{p}$ rounds.

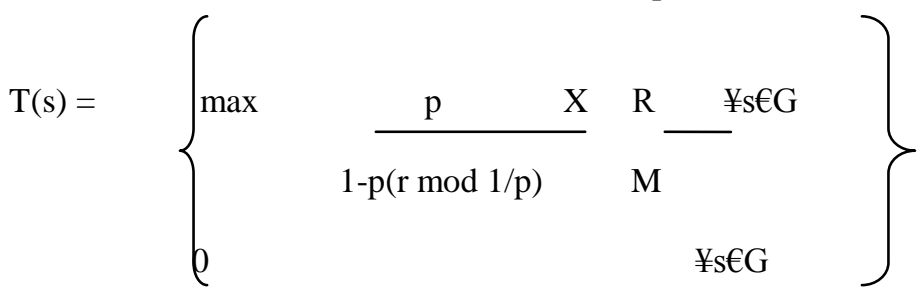

This is the formula used to calculate the threshold value for sensor node. Based upon the same formula in moLeach the threshold value is calculated for two rounds as follows:

$$
\mathrm{T}(\mathrm{s}) \mathrm{x}=\left\{\begin{array}{ccc}
\max \frac{\mathrm{p}}{\mathrm{RE} . . \mathrm{Tmin}} & \text { if } \mathrm{s} \in \mathrm{G} \\
1-\mathrm{p}(\mathrm{r} \bmod 1 / \mathrm{p}) & \mathrm{M}^{* 2} & \\
0 & & \text { otherwise }
\end{array}\right\}
$$

Where Tmin is minimum threshold energy, $M$ is maximum energy and RE is residual energy. If the node approve this formula then the node selected as a cluster head is capable to become cluster head for two rounds. Whereas if the same formula is not approved by node then another formula is used for determining the ability of node to become cluster head for one round.

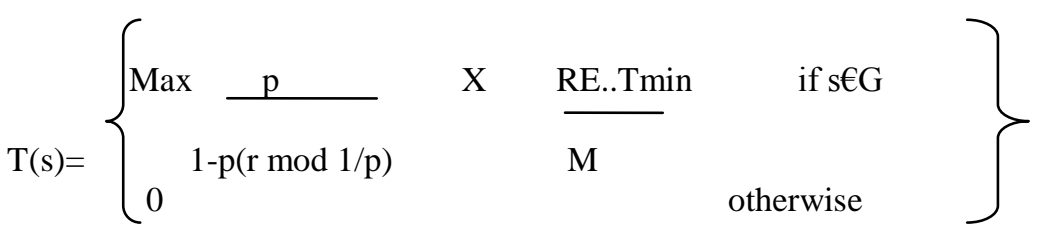

This is the approach used in selection of the cluster head in order to minimize the energy wastage in selecting the cluster head for next round .

Dual transmitting power levels:- In this scheme different power levels are used for different transmissions .Depending on the distance of the nodes, power levels are used for the amplification. Whereas in Leach same amplification energy is used for all transmissions. When the communication is within a cluster, means intra cluster communication. Where the data is sensed by cluster members and reported to cluster head. This is called intra cluster communication. The amplification energy required for intra cluster communication is low. Thus using low power level for intra cluster communication saves energy. 
When the communication is between two cluster heads, it is called inter cluster communication and there is one more communication that is cluster head to base station communication. The energy requirement cannot be same for all types of communication. Minimum energy is required for inter cluster communication. Whereas energy requiredfor cluster head to base station is high. In other clustering protocols same energy is used for communications. This scheme provides two type of power levels for communication one high power level and low power level. When the node which is used as a cluster head, routing protocol informs it to use high amplification energy. When the same node becomes cluster member in any other round routing protocol switches it to low amplification energy. So different levels of power are used in order to minimize the energy wastage as in other schemes by issuing same energy for different communication.

\subsection{Design Diagram}

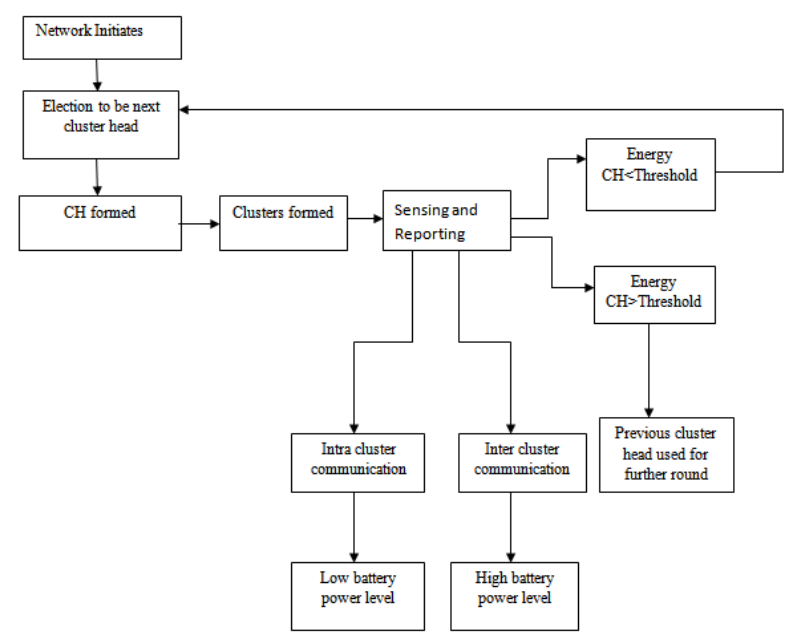

Fig. 2 Design Diagram

\section{Simulations}

Simulations are conducted in Matlab. In order to show the performance of ICHALEC specific intervals and parameters are taken. ICHALEC is compared with LEACH , MOD-LEACH in terms of network lifetime and it is clear from the graph that ICHALEC outperforms LEACH and MOD-LEACH.

\subsection{Simulation Scenario and Parameters}

In order to perform simulations a network size $100 \times 100 \mathrm{~m}^{2}$ is taken. Total nodes taken are 100 with initial energy of sensor nodes $0.5 \mathrm{~J}$ and packet size $4000 \mathrm{bits}$. Transceiver idle state energy consumption 50nj/bit with data fusion and aggregation energy consumption $5 \mathrm{nj} / \mathrm{bit}$.

\subsection{Simulation Results}

In a wireless sensor network computing capacity and stored energy is limited. Due to this limiting computing capacities, network lifetime and throughput is affected. Thus in the simulation the ICHEAC is evaluated using following indices.

\subsubsection{Network Life Time}

The network lifetime of the sensor network is the lifetime of the network from the starting of the network to the end of the network. It means the time from where the network starts its operation till the phase network has completed its operation. The operation is measured in terms of the rounds. Thus the network lifetime is measured in two ways alive nodes and dead nodes.

\subsubsection{Throughput}

The amount of data received by the base station describes the rate of the accuracy of the nodes, throughput. The more data received means high accuracy. The throughput of the sensor network is measured by the total number of packets sent to base station, packets sent to cluster head during the network lifetime and cluster head formation.

\subsection{Simulation Analysis}


The implementation of the ICHALEC is shown in form of graphs and the original LEACH and MOD-LEACH protocol is compared to the ICHALEC. Fig.3 and Fig.4 shows the change of alive nodes and dead nodes over the rounds. In Fig. 3 it is clear that the number of alive nodes are higher between 0 and 500 rounds. Further as the rounds are increasing ranging from 1000 to 1500, the number of alive nodes are decreasing. In fig 4.the number of dead nodes are shown over the rounds. It is clear that number of dead nodes are low in initial rounds ranging between 0 and 500. Between 1000 and 1500 rounds the range of dead nodes is higher. The change of alive nodes and dead nodes show the operational time of the ICHALEC protocol.

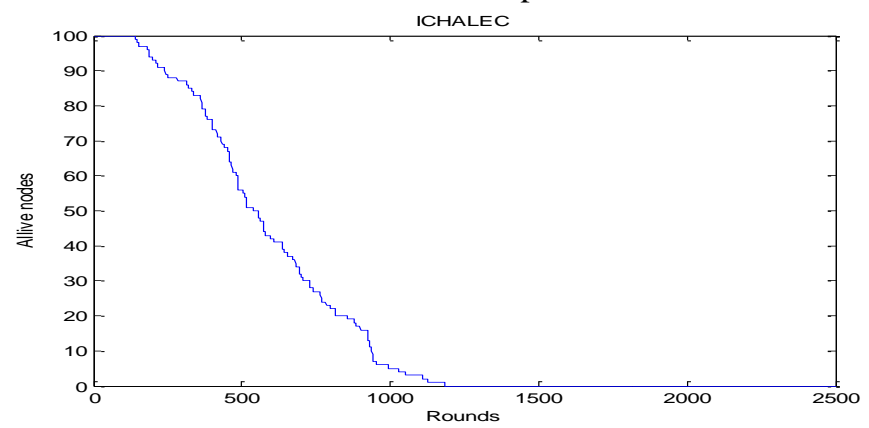

Fig.3 Alive nodes vs. Round

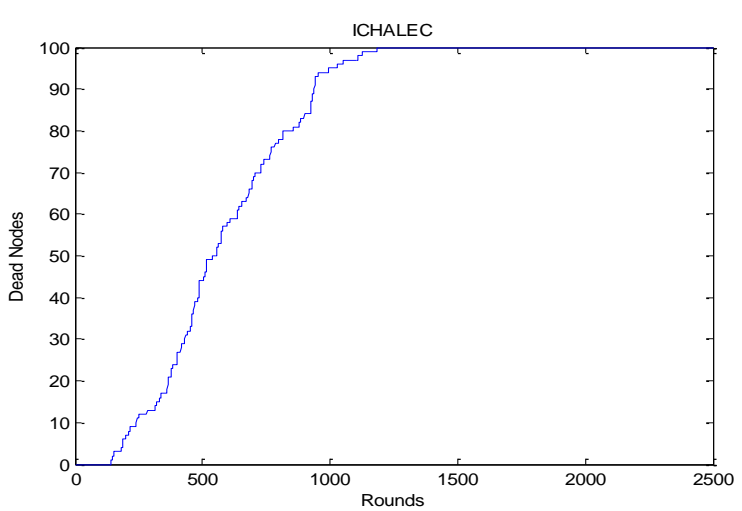

Fig.4 Dead nodes vs. Rounds

In Fig 5 and 6,the analysis of the number of packets sent to Base station and Cluster head is shown over rounds in order to measure accuracy. In fig 5 it is clear that the packets sent to base station increases as the number of rounds increases. More packets are sent to base station between 1500 and 2500 rounds.

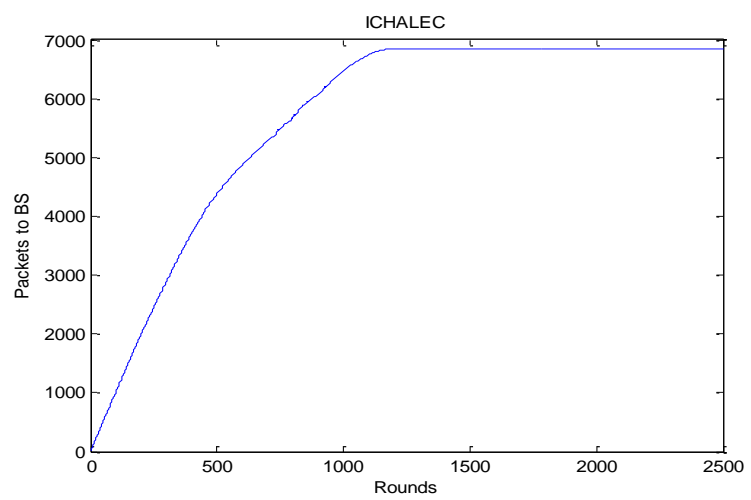

Fig . 5 packets to BS between Rounds 


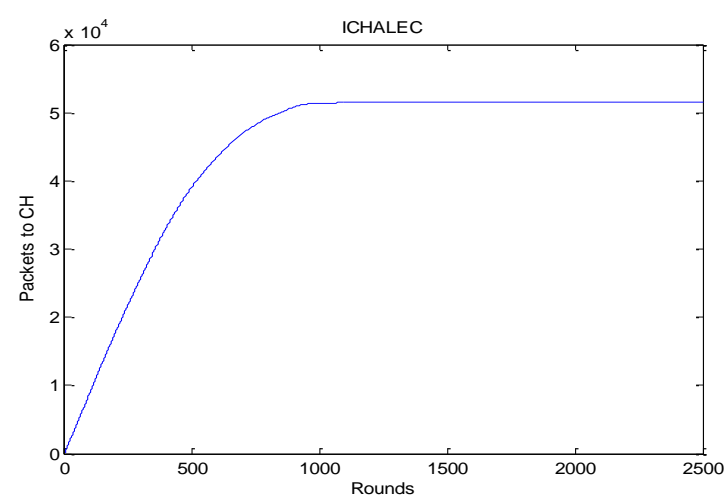

Fig 6. Packets to CH between rounds

In fig 6 the number of packets sent to the cluster head are shown. The number of packets sent to cluster head also increases as the number of rounds increases. The high number of data packets are sent between 1000 and 2500 rounds. The more the data packets sent to the cluster head the more accuracy of the network lifetime is achieved. This transmission shows the accuracy of the network life time. In fig 7 the cluster head formation is shown over rounds. The variation of the cluster head formation is shown between 0 and 1000 rounds.

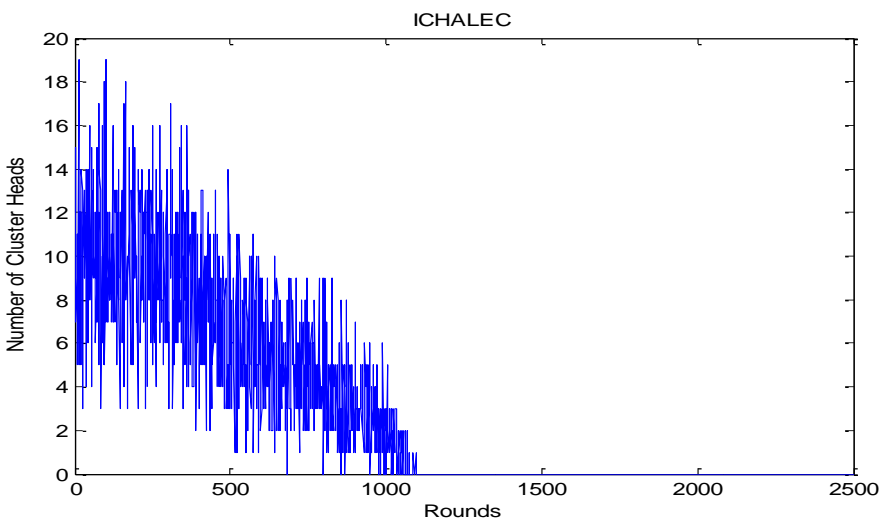

Fig.7.Number of Cluster head formed vs. rounds

In Fig.8 ICHALEC is compared with LEACH and MOD-Leach in terms of network life time and ICHALEC outperforms both the protocols .

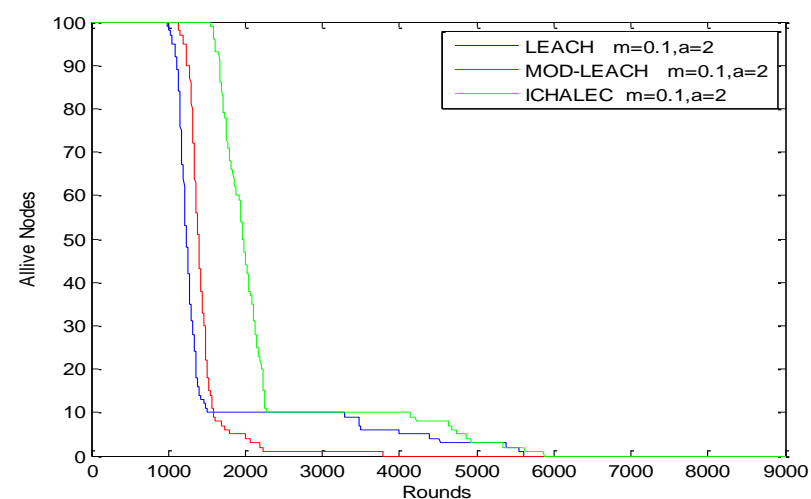

Fig8.Comparison of LEACH, MOD-LEACH and ICHALEC

\section{Conclusion and future work}

In this work new clustering approach is proposed ICHALEC, which can be further utilized in other clustering routing protocols for better efficiency. In this efficient cluster head replacement scheme is used and dual transmitting power level. Whenever cluster head is selected in first round then the same node can be used as a cluster head in other round if the energy of the node is greater then the threshold. In this way the energy of the cluster head is not wasted. If the energy of the cluster head is less than the threshold then the other node is chosen as a cluster head for next round. In this method the threshold is given priority for the choosing of the 
cluster head. And for different types of communication different power levels are used either low or high. Depending upon the distance the different amplification energy is used for the different types of communication.

Further the work on data aggregation and fusion can be carried out in cluster selection. Hence there is need to develop the new protocol which spends least energy in receiving and fusion of the data to base station and much more efficient mechanisms for the cluster head replacement .

\section{References}

[1]. P. Krishna, et al., “A survey on sensor networks", IEEE communications magazine, volume 8, pp 102-114, 2002.

[2]. M. Akbar, N. Javaid, A. A. Khan, Z. A. Khan, U. Qasim, "On Modeling Geometric Joint Sink Mobility with Delay-tolerant Clusterless Wireless Sensor Networks", 4th IEEE Technically Co-Sponsored International Conference on Smart Communications in Network Technologies (SaCoNet'13) 2013, Paris, France.

[3]. V. Mhatre, et al., "Design of surveillance sensor grids with a lifetime constraint", 1st European Workshop on Wireless Sensor Networks (EWSN), Berlin, January 2004.

[4]. W.R. Heinzelman, et al., "Energy efficient communication protocol for wireless micro sensor networks", Proceedings of the 33rd Hawaii International Conference on System Sciences (HICSS-33), January 2000.

[5] W. Heinzelman, A. Chandrakasan, and H. Balakrishnan."Energy-Efficient Communication Protocols for Wireless Microsensor Networks".In Proceedings of Hawaiian International Conference on Systems Science, January 2000.

[6] C. Intanagonwiwat, R. Govindan, and D. Estrin.”Directed Diffusion: AScalable and Robust Communication Paradigm for Sensor Networks".In Proceedings of the 6th Annual ACM/IEEE International Conferenceon Mobile Computing and Networking(MOBICOM), pp. 56-67, August2000.

[7] D. Estrin, R. Govindan, J. Heidemann, and S. Kumar. "Next Century Challenges: Scalable Coordination inWireless Networks". In Proceedings of the 5th Annual ACM/IEEE International Conference on Mobile Computing and Networking( MOBICOM), pp. 263$270,1999$.

[8]. Sze-yao Ni, et al., "The broadcast storm problem in a mobile Ad Hoc network", Proceedings of MobiCom, August 1999.

[9]. D. Estrin, et al., "Next century challenges: scalable coordination in sensor networks", MobiCom, August 1999.

[10] M. Tahir, N. Javaid, Z. A. Khan, U. Qasim and M. Ishfaq, "EAST: Energy-Efficient Adaptive Scheme for Transmission In Wireless Sensor Networks", 26th IEEE Canadian Conference on Electrical and Computer Engineering (CCECE2013), Regina, Saskatchewan, Canada, 2013.

[11] Smaragdakis G, Matta I, Bestavros A. "SEP: A stable election protocol for clustered heterogeneous wireless sensor networks", Proc of the Int'1 Workshop on SANPA 2004. pp. 251-261, 2004.

[12]. W.R. Heinzelman, et al., "Energy efficient communication protocol for wireless micro sensor networks", Proceedings of the 33rd Hawaii International Conference on System Sciences (HICSS-33), January 2000.

[13] B. Manzoor, N. Javaid, O. Rehman, M. Akbar, Q. Nadeem, A. Iqbal, M. Ishfaq, "Q-LEACH: A New Routing Protocol for WSNs", International Workshop on Body Area Sensor Networks (BASNet-2013) in conjunction with 4th International Conference on Ambient Systems, Networks and Technologies (ANT 2013), 2013, Halifax, Nova Scotia, Canada, Procedia Computer Science, Volume 19, 2013, Pages 926-931, ISSN 1877-0509.

[14] Mona El_Saadawy,et al, “Enhancing S-LEACH Security for Wireless Sensor Networks,” IEEE 2012 .

[15] JiaXu,et al, "Improvement of LEACH protocol for WSN," 2012 IEEE.

[16]. W.R. Heinzelman, et al., "Energy efficient communication protocol for wireless micro sensor networks", Proceedings of the 33rd Hawaii International Conference on System Sciences (HICSS-33), January 2000. 